\title{
Success rate of epicardial left atrial ablation during coronary artery bypass grafting and aortic valve replacement: A pilot single centre study
}

Mohamed Salem*\#, Alexander Thiem*\#, Jan Dreyer, Christina Grothusen, Thomas Puehler, Markus Ernst, Christine Friedrich, Assad Haneya, Jochen Cremer and Jan Schöttler

${ }^{1}$ Department of Cardiovascular Surgery, University Hospital of Kiel, Schleswig-Holstein, Germany

\#Both authors contribute equally to this work.

\begin{abstract}
Objective: The incidence of atrial fibrillation is increasing continuously. The impact of the underlying cardiac disease on success rate of epicardial left atrial ablation after cardiac surgery is not consistently investigated. Success rate of epicardial left atrial ablation after cardiac surgery were analysed in 30 patients, who intraoperatively received an event recorder. Therapeutic success was evaluated one year postoperatively. Furthermore, long-term survival after five years was investigated. Patients with isolated coronary artery bypass graft $(n=17)$ were compared to patients with aortic valve replacement with or without combination with coronary artery bypass graft $(\mathrm{n}=13)$.

Results: There was no significant difference between both groups in demographic, intraoperative and postoperative variables. One year after aortic valve replacement, the proportion of patients with a stable sinus rhythm was significantly lower than after coronary artery bypass grafting only $(23.1 \%$ vs. $64.7 \%$, $p=0.033)$. If preoperatively paroxysmal atrial fibrillation was diagnosed, the success rate after coronary artery bypass grafting (77.8\%) as well as aortic valve replacement (25.0\%) were higher. Five-year survival rate after isolated coronary artery bypass grafting was $82.4 \%$ and after aortic valve replacement was $100 \%$.

Conclusions: Epicardial left atrial ablation is a good treatment option for patients with atrial fibrillation undergoing an isolated CABG. Due to the low chance of success in patients undergoing isolated aortic valve replacement or combined intervention with CABG, the epicardial ablation rather not to be performed. Fortunately, a satisfying overall five-year survival rate could be seen.
\end{abstract}

Abbreviations: SD: Standard Deviation; CABG: Coronary Artery Bypass Grafting; AVR: Aortic Valve Replacement; LVEF: Left Ventricular Ejection Fraction; ICU: Intensive Care Unit

\section{Introduction}

\section{Objective}

Worldwide, the incidence of atrial fibrillation is continuously increasing. Epidemiological analyses in Germany showed that at least 400 per 100.000 people suffered from atrial fibrillation in 2010 [1]. The long-term prognosis is low due to high risk of strokes and mortality associated with atrial fibrillation [2]. Risk factors for atrial fibrillation are numerous. Valvular heart disease and coronary artery disease are considered common comorbidities [3]. The impact of the underlying cardiac disease on the success rate of epicardial left atrial ablation during cardiac surgery is not clear.

In the current analysis we compare two groups of patients suffered from atrial fibrillation who received an easy, standardised, epicardial left atrial ablation and implantation of event recorder during isolated coronary artery bypass grafting (CABG) versus isolated aortic valve replacement or a combined intervention (aortic valve replacement plus $\mathrm{CABG}$ ). The study focusses on the success rates of the ablation procedure after one year as well as the long-term survival after five years.

\section{Methods}

\section{Patients}

As shown in figure 1 , this is a retrospective analysis included 49 patients, who received an epicardial left atrial ultrasound ablation, as well as an implantation of an event recorder during CABG or an aortic valve replacement, or a combination of both in our hospital between October 2009 and July 2012. Exclusion criteria were cardiac reoperation, other cardiac operations and combined interventions or a preoperatively implanted pacemaker. Final inclusion criterion was a completed one-year follow-up. The reasons for incompletion of one-year follow-up in 19 patients are shown in figure 1. A cohort of 30 patients were included, who gave their written informed consent

${ }^{\star}$ Correspondence to: Mohamed Salem, Department of Cardiovascular Surgery, University Hospital of Kiel, Schleswig-Holstein, Germany, Arnold-Heller-Str. 3 Haus C, 24105 Kiel, Germany Tel: +49 (0) 431 50022006; Fax: +49 (0) 431 50022004; E-mail: Mohamed.salem@uksh.de

Alexander Thiem, Department of cardiovascular surgery University Hospital of Kiel, Schleswig-Holstein, Germany, E-mail: Alexander.Thiem@uksh.de

Key words: atrial fibrillation, coronary artery bypass grafting, aortic valve replacement, surgical pulmonary vein isolation, event recorder

Received: October 10, 2020; Accepted: October 27, 2020; Published: October 30,2020 


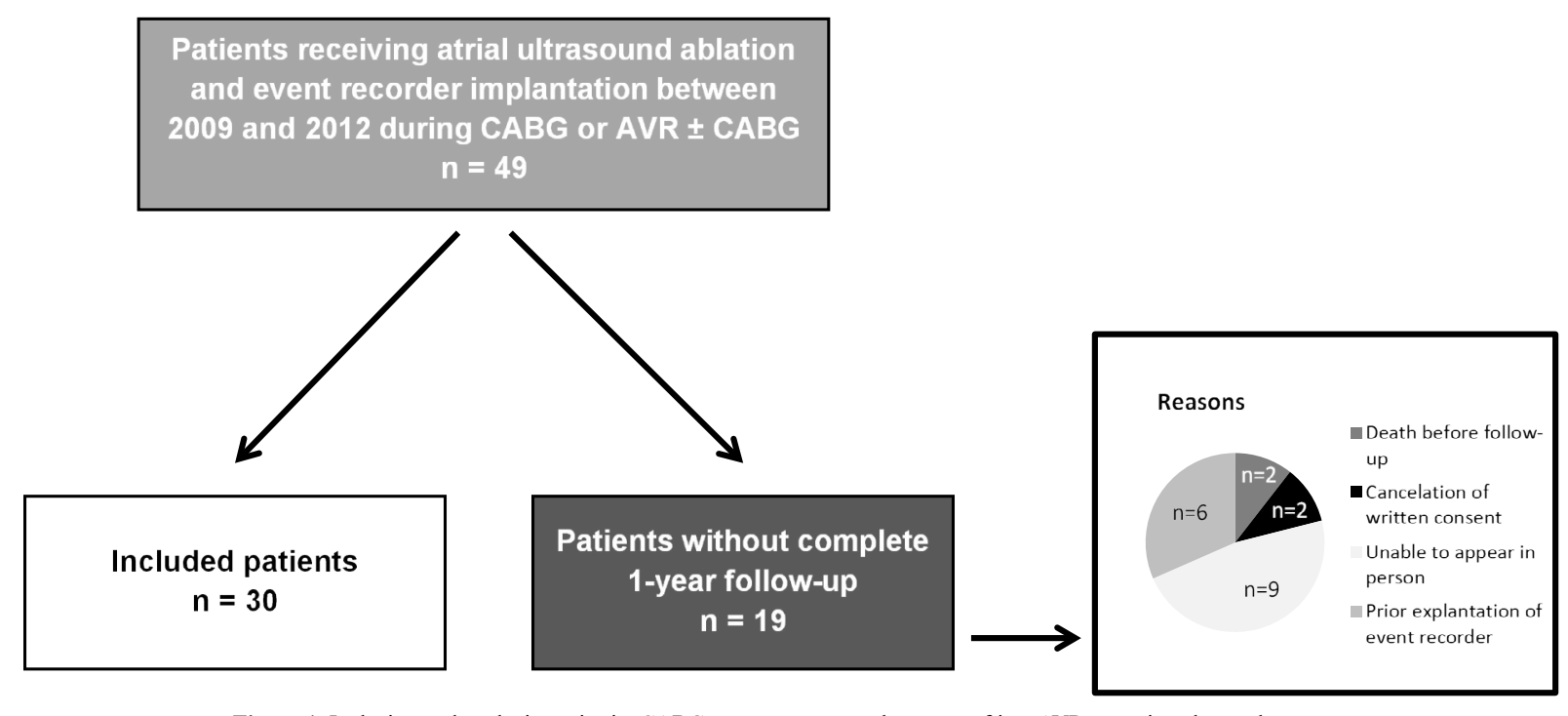

Figure 1. Inclusion and exclusion criteria, $\mathrm{CABG}=$ coronary artery bypass grafting, $\mathrm{AVR}=$ aortic valve replacement.

for using their patient data for the purposes of research at the time of admission to our hospital. The approval for this study was obtained by the local ethics committee (D473/13).

\begin{abstract}
Ablation
The left atrial ultrasound ablation was performed by using the Epicor ablation system (St. Jude Medical). With this system a standardised box lesion of the left atrial posterior wall including the pulmonary veins was induced (Figure 2). For this purpose, the Epicor tool was guided through the transverse pericardial sinus above the left pulmonary veins and led back through the oblique sinus to be merged above the right pulmonary veins. No other ablation lines were set by other tools; the left atrial appendage was not occluded or resected. The ablations were performed on cardiopulmonary bypass on the beating heart before aortic cross-clamping.
\end{abstract}

\section{Event recorder}

An Event recorder Reveal XT (Medtronic) was implanted in all patients in the subcutaneous tissue in a left parasternal position. The devices were activated postoperatively before hospital discharge. At the time of interrogation of the device at follow-up, the atrial fibrillation burden is displayed as a percentage value. This value shows the duration of atrial fibrillation episodes in proportion to the total time of rhythm analysis.

The sensitivity of the event recorder in detecting atrial fibrillation is $96.1 \%$, specificity is $85.4 \%$, the positive predictive value is $79.3 \%$ and the negative predictive value is $97.4 \%$, according to results of the XPECT-Study [4].

\section{One-year follow-up}

All included patients received an interrogation of the event recorder at one-year follow-up. In addition, a standard 12-lead ECG was recorded. A successful ablation therapy was assumed, if the atrial fibrillation burden was less than $0.5 \%$ since the operation, and if, in addition, a sinus rhythm was documented in the 12-lead ECG.

\section{Long-term survival}

For investigation of long-term survival, patients were called, or the local civil registration offices were contacted.

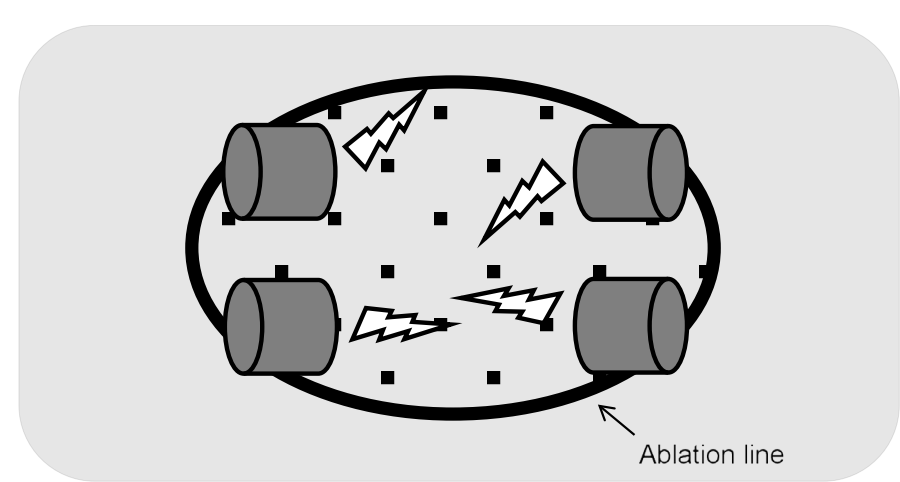

Figure 2. Schematic image of ablation line enclosing all pulmonary vein ostia (so called "box lesion").

\section{Statistics}

Patient characteristics were presented as mean and standard deviation and compared by unpaired t-test for approximately normally distributed continuous variables. Not-normally distributed continuous data were compared by Mann-Whitney $U$ test. Categorial data were described as absolute (n) and relative (\%) frequencies and compared by Fisher's exact test.

Survival was calculated on right-censored data by Kaplan-Meier Analysis and compared by log rank test. All statistical tests were conducted two-tailed with a significance level of 5\%. Data were analyzed with IBM SPSS Statistics for Windows (Version 23.0).

\section{Results}

All 30 patients were surveyed retrospectively. Seventeen patients had received atrial ablation during isolated coronary artery bypass grafting, 13 patients during aortic valve replacement, 6 of these in combination with CABG.

Preoperative demographic data is shown in table 1. Both groups had a mean age of slightly above 70 years $(71.3 \pm 4.9$ years vs. 71.7 \pm 8.2 years). The male gender distribution was similar represents $82.4 \%$ vs. $84.6 \%$. The incidence of atrial fibrillation, the percentages of patients with a low left ventricular ejection fraction preoperatively and the $\mathrm{CHA}^{2} \mathrm{DS}^{2}$-VASc-Scores did not significantly differ between 
both groups. In each of the two groups, one patient had a previous neurological event in his medical history.

In table 2, the average operating time, cardiopulmonary bypass time, and cross-clamp time are shown. Operating time and cardiopulmonary bypass time did not significantly vary between the groups. The aortic cross-clamp time was significantly shorter during isolated coronary artery bypass grafting $(71 \pm 21 \mathrm{~min}$ vs. $95 \pm 33 \mathrm{~min} ; \mathrm{p}=0.019)$.

Table 3 shows data concerning the postoperative hospital course. Duration of treatment on the intensive care unit and duration of hospitalisation were comparable. While no patient in the isolated coronary artery bypass grafting group required a pacemaker implantation during postoperative hospital stay, there was one patient in the aortic valve replacement group who received a permanent pacemaker. Two patients in the aortic valve replacement group suffered from perioperative neurological events.

One-year follow-up data is demonstrated in table 4. Until followup one coronary patient received a pacemaker implantation. In both groups, no further neurological events were seen after hospital discharge. Regarding the success rate of atrial ablation after one year,

Table 1. Patient characteristics, age at the time of operation and $\mathrm{CHA}^{2} \mathrm{DS}^{2} \mathrm{Vasc}-\mathrm{SCORE}$ are shown as mean values and standard deviation $(\mathrm{SD}) . \mathrm{CABG}=$ coronary artery bypass grafting, $\mathrm{AVR}=$ aortic valve replacement, $\mathrm{LVEF}=$ left ventricular ejection fraction .

\begin{tabular}{|l|c|c|c|}
\hline \multicolumn{1}{|c|}{} & CABG only & AVR \pm CABG & p-value \\
\hline Number of patients & 17 & 13 & \\
\hline Age [years] & $71.3 \pm 4.9$ & $71.7 \pm 8.2$ & 0.878 \\
\hline Male gender & $14(82.4 \%)$ & $11(84.6 \%)$ & 1.000 \\
\hline Paroxysmal atrial fibrillation & $9(52.9 \%)$ & $4(30.8 \%)$ & 0.283 \\
\hline Non-paroxysmal atrial fibrillation & $8(47.1 \%)$ & $9(69.2 \%)$ & 0.283 \\
\hline Persistent atrial fibrillation & $6(35.3 \%)$ & $4(30.8 \%)$ & 1.000 \\
\hline Long-standing persistent atrial fibrillation & $2(11.8 \%)$ & $5(38.5 \%)$ & 0.190 \\
\hline Atrial fibrillation at admission & $6(35.3 \%)$ & $7(53.8 \%)$ & 0.460 \\
\hline CHA ${ }^{2} D S^{2}$ Vasc-SCORE & $3.1 \pm 1.2$ & $3.1 \pm 1.4$ & 0.934 \\
\hline LVEF $<50 \%$ & $2(11.8 \%)$ & $5(38.5 \%)$ & 0.190 \\
\hline Neurological event in medical history & $1(5.9 \%)$ & $1(7.7 \%)$ & 1.000 \\
\hline
\end{tabular}

Table 2. Intraoperative variables, operation times in minutes are shown as mean values and standard deviation $(\mathrm{SD}), \mathrm{CABG}=$ coronary artery bypass grafting, $\mathrm{AVR}=$ aortic valve replacement.

\begin{tabular}{|l|c|c|c|}
\hline & CABG only & AVR \pm CABG & p-value \\
\hline Duration of operation [min] & $275 \pm 49$ & $266 \pm 60$ & 0.667 \\
\hline Bypass time [min] & $126 \pm 31$ & $150 \pm 45$ & 0.090 \\
\hline Cross clamp time [min] & $71 \pm 21$ & $95 \pm 33$ & $\mathbf{0 . 0 1 9}$ \\
\hline
\end{tabular}

Table 3. Postoperative variables, length of ICU stay and length of hospital stay are shown as mean values and standard deviation $(\mathrm{SD}), \mathrm{CABG}=$ coronary artery bypass grafting, $\mathrm{AVR}$ $=$ aortic valve replacement, ICU $=$ Intensive Care Unit.

\begin{tabular}{|l|c|c|c|}
\hline & CABG only & AVR \pm CABG & p-value \\
\hline Length of ICU stay [days] & $2.8 \pm 1.7$ & $2.1 \pm 0.3$ & 0.432 \\
\hline Length of hospital stay [days] & $9.8 \pm 3.9$ & $9.3 \pm 4.3$ & 0.765 \\
\hline Pacemaker implantations [\%] & $0(0 \%)$ & $1(7.7 \%)$ & 0.433 \\
\hline Sinus rhythm at hospital discharge [\%] & $11(64.7 \%)$ & $5(38.5 \%)$ & 0.269 \\
\hline Neurological events [\%] & $0(0 \%)$ & $2(15.4 \%)$ & 0.179 \\
\hline
\end{tabular}

Table 4. One-year follow-up data, $\mathrm{CABG}=$ coronary artery bypass grafting, $\mathrm{AVR}=$ aortic valve replacement.

\begin{tabular}{|l|c|c|c|}
\hline & CABG only & AVR \pm CABG & p-value \\
\hline Sinus rhythm and Burden $<0.5 \%$ & $11(64.7 \%)$ & $3(23.1 \%)$ & $\mathbf{0 . 0 3 3}$ \\
\hline $\begin{array}{l}\text { Pacemaker implantations after hospital } \\
\text { discharge [\%] }\end{array}$ & $1(5.9 \%)$ & $0(0 \%)$ & 1.000 \\
\hline $\begin{array}{l}\text { Neurological events after hospital } \\
\text { discharge [\%] }\end{array}$ & $0(0 \%)$ & $0(0 \%)$ & 1.000 \\
\hline
\end{tabular}

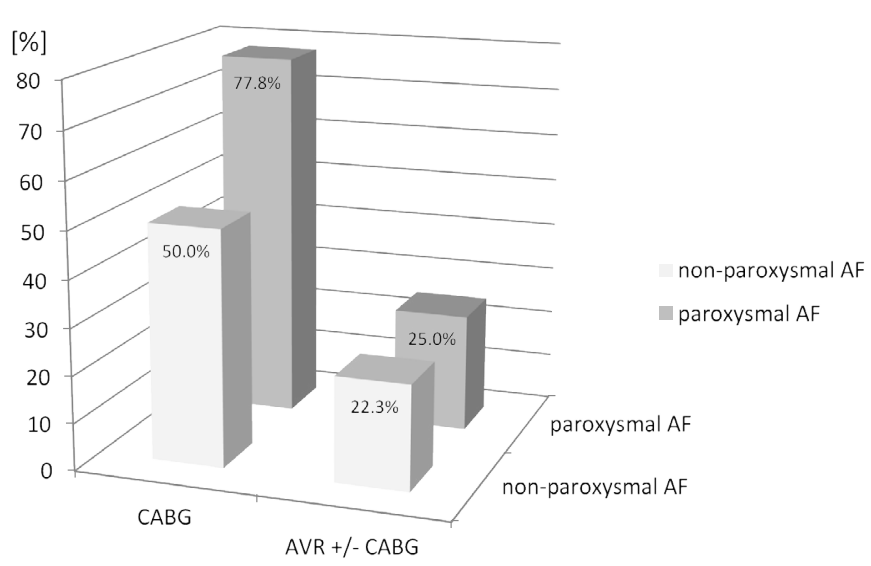

Figure 3. Success rates in \% in relation to the type of operation and type of atrial fibrillation, $\mathrm{CABG}=$ coronary artery bypass grafting, $\mathrm{AVR}=$ aortic valve replacement, $\mathrm{AF}=$ atrial fibrillation.

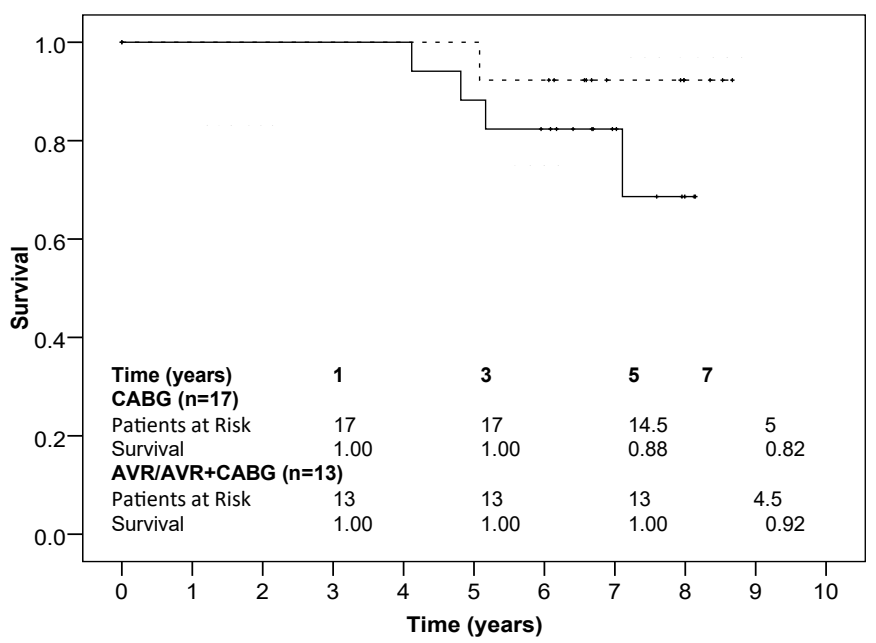

Figure 4. Kaplan-Meier-survival after isolated coronary artery bypass grafting and after aortic valve replacement procedures, $\mathrm{CABG}=$ coronary artery bypass graft, $\mathrm{AVR}=$ aortic valve replacement.

the highest rate with $64.7 \%(11 / 17)$ was seen in the group of patients after isolated coronary artery grafting. After aortic valve replacement the treatment of atrial ablation was only successful in $23.1 \%(3 / 13)$ $(\mathrm{p}=0.033)$. The success rate after isolated aortic valve replacement was not significantly better when compared to combined interventions (28.6\% vs. $16.7 \%)$.

Higher rates of atrial ablation success were observed in patients with preoperative paroxysmal atrial fibrillation after both isolated coronary artery bypass grafting and aortic valve replacement $[77.8 \%$ (7/9) and 25.0\% (1/4)] (Figure 3).

There was no significant difference in five-year survival after isolated coronary artery bypass grafting and after aortic valve replacement. Overall, only three patients died within five years after coronary artery bypass grafting, in which the ablation procedure was successful in two of them at the time of one-year follow-up. A Kaplan-Meier graph showing the long-term survival in figure 4.

\section{Discussion}

Patients with pre-existing atrial fibrillation undergoing cardiac surgery have a poor prognosis compared to patients with a stable sinus 
rhythm [5,6]. Nowadays, simultaneous epicardial atrial ablation can be performed during cardiac surgery with a low risk. Only a few working groups apply the complete Cox-MAZE IV procedure [7]. In most centres, especially during coronary or aortic valve surgery, simplified ablation procedures are performed [8-11].

A meta-analysis by McClure et al. published in 2017 showed the main problem in the scientific approach to atrial ablation during cardiac surgery. In publications on this subject there is often not only a difference in ablation techniques, but also regarding the localisation of the ablation lines [12]. These factors can of course have an influence on the success of atrial ablation and on the comparability of the results.

Another problem is the inaccuracy of postoperative evaluation of success of the procedure. Conventional 12-lead ECGs but also 24-, 48- or 72-hour long-term ECG show a low sensitivity in detecting especially paroxysmal atrial fibrillation. In 2009 Hanke et al. showed, that even 24-hour ECGs recorded quarterly after surgical atrial ablation had a sensitivity of only $60 \%$ and a negative predictive value of only $64 \%$ regarding atrial fibrillation [13]. We believe that success rates after atrial ablation, based on this type of follow-up examinations only, should be evaluated critically, in contrast to surveys based on continuous long-term rhythm monitoring over months or years using an event recorder.

To make simultaneous atrial ablation available for every patient undergoing cardiac surgery in our centre, independent of the operating surgeon, as well as to achieve a precise analysis based on long-term follow-up, we started to standardize the procedure in 2009. Every patient with pre-existing atrial fibrillation, who underwent cardiac surgery in our centre between October 2009 and July 2012 and agreed to the procedure, received a standardised atrial ablation using the Epicor ${ }^{\circledR}$ ablation system by St. Jude Medical. During this period only, patients undergoing mitral valve surgery were treated with other tools, primarily performing endoatrial ablations.

Knaut et al. could show in 2010 that patients with pre-existing permanent atrial fibrillation undergoing cardiac surgery because of an aortic valve stenosis or coronary artery disease (or both) benefit from a simultaneous atrial ablation regarding restoration of sinus rhythm postoperatively [10]. The impact of the underlying cardiac disease on success of atrial ablation stayed unclear, not only in this exemplarily named publication. In our study the success rates of atrial ablation during coronary artery bypass grafting or aortic valve replacements (or both) were directly compared for the first time.

An ablation procedure was seen as successful, if atrial fibrillation burden was below $0.5 \%$ until interrogation of the event recorder at the time of one-year follow-up and if a sinus rhythm could be documented at that point. In their publication from 2012, Pokushalov et al. analysed the benefit of atrial ablation during coronary artery bypass grafting in patients with recently diagnosed atrial fibrillation. Also using the Reveal XT" event recorder an atrial fibrillation burden below $0.5 \%$ was seen as success of the ablation procedure.

After all we have knowledge of only a few studies, which used an event recorder for success evaluation after surgical ablation $[8,9,13]$.

In the current analysis, the best success rate was seen in patients after coronary artery bypass grafting only with $64.7 \%$. This success rate was significantly higher than in patients after aortic valve replacement with $23.1 \%$. The highest recurrence rate was seen in patients after combined interventions (aortic valve replacement plus coronary artery bypass grafting). In these patients the success rate was only $16.7 \%$. The subanalysis of patients with paroxysmal atrial fibrillation showed better results in both groups, in which the success rate in patients with CABG was $77.8 \%$ vs. $25.0 \%$ in patients with aortic valve replacement. Pukushalov et al. even saw a success rate of $89 \%$ of patients with coronary artery disease 18 months after epicardial pulmonary vein isolation using radio frequency ablation [9].

According to our results the applied ablation method can be recommended for patients undergoing coronary artery bypass grafting and especially for treatment of paroxysmal atrial fibrillation. For patients with aortic valve stenosis the described technique cannot be recommended due to its poor success rate, even if only paroxysmal atrial fibrillation was diagnosed. Success rates were even worse in patients with non-paroxysmal atrial fibrillation, and in general in patients undergoing combined interventions.

Probably these patients need a more potent ablation procedure, eventually by selecting additional ablation lines, using different thermal energy sources or performing a bi-atrial ablation. Appropriate modifications of the procedure maybe be found with better results. Under circumstances for these patients a two-step hybrid ablation might be a good option [14].

Even if in this study no patient suffered from a stroke between hospital discharge and the one-year follow-up, it should be discussed whether an ablation therapy should be combined with occlusion of the left atrial appendage, optionally by clipping [15].

Long-term survival was encouragingly high in our study. The poor success of atrial ablation in patients undergoing aortic valve replacement did not have a negative effect on long-term survival in our study group. On the contrary, a study from Schulenberg and co-workers showed an impaired long-term outcome of patients with preoperatively diagnosed atrial fibrillation after aortic valve replacement alone or in combination with myocardial revascularization compared to patients without a history of atrial fibrillation [16].

\section{Study limitation}

The results of this study are limited due to the retrospective analyses of only those patients who agreed to be examined at one-year followup as well as to receive an event recorder implantation. Therefore, we cannot give detailed information about the morbidity and mortality of other patients who were treated in the same way during the period of investigation. Another disadvantage of this investigation is the small number of patients included. On the other hand, we underline the clear method and strict definition of the success of therapy.

\section{Conclusion}

Epicardial ablation of the left atrial posterior wall including the pulmonary veins during cardiac surgery is a simple, easy to learn method with only few complications. Performed during coronary artery bypass grafting, this method shows good results for patients with paroxysmal atrial fibrillation and still acceptable results for patients with non-paroxysmal atrial fibrillation. For patients with aortic valve replacement with or without combination of CABG, more successful method has to be developed yet.

\section{References}

1. Chugh SS, Havmoeller R, Narayanan K, Singh D, Rienstra M, et al. (2014) Worldwide Epidemiology of Atrial Fibrillation: A Global Burden of Disease 2010 Study. Circulation 129: 837-847. [Crossref]

2. Kannel WB, Wolf PA, Benjamin EJ, Levy D (1998) Prevalence, incidence, prognosis, and prediposing conditions for atrial fibrillation: population-based estimates. $\mathrm{Am} \mathrm{J}$ Cardiol 82(8a): 2N-9N. [Crossref] 
3. Naser N, Dilic M, Durak A, Kulic M, Pepic E, et al. (2017) The Impact of Risk Factors and Comorbidities on The Incidence of Atrial Fibrillation. Mater Sociomed 29: 231236. [Crossref]

4. Hindricks G, Pokushalov E, Urban L, Taborsky M, Kuck KH, et al. (2010) Performance of a New Leadless Implatable cardiac Monitor in Detecting and Quantifying Atrial Fibrillation Results of the XPECT Trial. Circ Arrhythm Electrophysiol 3: 141-147. [Crossref]

5. Ad N, Barnett SD, Haan CK, O’Brien SM, Milford-Beland S, Speir AM (2009) Does preoperative atrial fibrillation increase the risk for mortality and morbidity after coronary artery bypass grafting? J Thorac Cardiovasc Surg 137: 901-906. [Crossref]

6. Thourani VH, Forcillo J, Szeto WY, Kodali SK, Blackstone EH, et al. (2018) Outcomes in 937 Intermediate-Risk Patients Undergoing Surgical Aortic Valve Replacement in PARTNER 2A. Ann Thorac Surg 105: 1322-1329. [Crossref]

7. Gaynor SL, Diodato MD, Prasad SM, Ishii Y, Schuessler RB, et al. (2004) A prospective, single-center clinical trial of a modified Cox maze procedure with bipolar radiofrequency ablation. J Thorac Cardiovasc Surg 128: 535-542. [Crossref]

8. Cherniavsky A, Kareva Y, Pak I, Rakhmonov S, Pokushalov E, et al. (2014) Assessment of results of surgical treatment for persistent atrial fibrillation during coronary artery bypass grafting using implantable loop recorders. Interact Cardiovasc Thorac Surg 18: 727-731. [Crossref]

9. Pokushalov E, Romanov A, Corbucci G, Cherniavsky A, Karaskov A (2012) Benefit of ablation of first diagnosed paroxysmal atrial fibrillation during coronary artery bypass grafting: a pilot study. Eur J cardiothorac Surg 41: 556-560. [Crossref]
10. Knaut M, Kolberg S, Brose S, Jung F (2013) Epicardial microwave ablation of permanent atrial fibrillation during a coronary bypass and/or aortic valve operation: Prospective, randomized, controlled, mono-centric study. Pathophysiol 17: 1-19. [Crossref]

11. Van Breugel HN, Nieman FH, Accord RE, Van Mastrigt GA, Nijs JF, et al. (2010) A prospective randomized multicenter comparison on health-related quality of life: The value of add-on arrhythmia surgery in patients with paroxysmal, permanent or persistent atrial fibrillation undergoing valvular and/or coronary bypass surgery. $J$ cardiovasc Electrophysiol 21: 511-520. [Crossref]

12. McClure GR, Belley-Cote EP, Jaffer IH, Dvirnik N, An KR, et al. (2017) Surgical ablation of atrial fibrillation: a systematic review and meta-analysis of randomized controlled trials. Europace 20: 1442-1450. [Crossref]

13. Hanke T, Charitos EI, Stierle U, Karluss A, Kraatz E, et al. (2009) Twenty-Four-Hour Holter Montor Follow-Up Does Not Provide Accurate Heart Rhythm Status After Surgical Atrial Fibrillation Ablation Therapy. Circulation 120: 177-184. [Crossref]

14. Kurfirst V, Mokracek A, Bulava A, Canadyova J, Hanis J, et al. (2014) Two-staged hybrid treatment of persistent atrial fibrillation: short-term single-centre results. Interact Cardiovasc Thorac Surg 18: 451-456. [Crossref]

15. Caliskan E, Sahin A, Yilmaz M, Seifert B, Hinzpeter R, et al. (2017) Epicardial lef atrial appendage AtriClip occlusion reduces the incidence of stroke in patients with atrial fibrillation undergoing cardiac surgery. Europace 20: e105-e114. [Crossref]

16. Schulenberg R, Antonitsis P, Stroebel A, Westaby S (2010) Chronic Atrial Fibrillation Is Associated with Reduced Survival After Aortic and Double Valve Replacement. Ann Thorac Surg 89: 738-744. [Crossref]

Copyright: (C2020 Salem M. This is an open-access article distributed under the terms of the Creative Commons Attribution License, which permits unrestricted use, distribution, and reproduction in any medium, provided the original author and source are credited. 